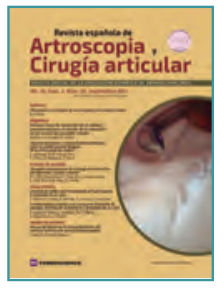

\title{
Original
}

\section{Primeras fases del desarrollo de un modelo experimental para el estudio de la reparación de las roturas del manguito rotador}

\author{
G. Busto, A. Jáuregui, R. Cuéllar \\ Instituto de Investigación Sanitaria Biodonostia. Donostia-San Sebastián, Gipuzkoa
}

Correspondencia:

Dr. Ricardo Cuéllar Gutiérrez

Correo electrónico: ricuellarg@gmail.com
Recibido el 9 de octubre de 2016

Aceptado el 13 de mayo de 2017

Disponible en Internet: septiembre de 2017

\section{RESUMEN}

Objetivo: Desarrollar un modelo animal de desgarro crónico del manguito rotador y crear un patrón crónico de características semejantes al humano.

Material y método: Ensayo aleatorizado, con 40 ratas Wistar-Ham, de 200-300 gramos. Se intervinieron ambas extremidades anteriores, según 2 grupos: a) con técnica simple de desinserción tendinosa (TS); y b) añadiendo la denervación del nervio supraescapular (TD). Efectuamos un estudio macro- y microscópico, a las 4, 8 y 12 semanas. El lado contralateral sirvió como control.

Resultados: Tanto la TS como la TD produjeron peso y volumen del supraespinoso (SE) e infraespinoso (IE) inferiores a los de los hombros control. Este efecto se mantuvo en el tiempo. El infiltrado graso muscular aumentó con el tiempo y fue mayor a las 12 semanas mediante la TD en los músculos $\mathrm{SE}(p<0,004)$ e IE $(p<0,02)$ que en los controles. No encontramos diferencias significativas en los parámetros tendinosos. Conclusiones: Los cambios provocados en las propiedades histológicas musculares a lo largo del presente trabajo son los propios de un modelo crónico de desgarro del manguito rotador. Representan un modelo fiable, reproducible y mantenido que permite el estudio de diferentes terapias biológicas coadyuvantes, en animal pequeño. El efecto de la denervación añade un mayor infiltrado graso muscular, aunque

\section{ABSTRACT \\ First stages of the development of an experimental model for the study of repair of rotator cuff tears}

Aim: To develop an animal model of chronic rotator cuff tear. To induce a chronic pattern similar to human being.

Methods: A randomized with 40 Wistar-Ham rats 200300 grams. Both forelimbs in 2 groups were involved: a) simple technique of tendon detachment (ST); b) technique in which suprascapular nerve denervation (DT) was added. At 4, 8 and 12 weeks were made macro- and microscopic study. The contralateral side served as control.

Results: Both the TS and TD weight and volume produced supraspinatus (SE) and infraspinatus (IE) below the shoulder control. This effect was maintained over time. Muscle fatty infiltration increased over time and was higher at 12 weeks by TD in the muscles SE ( $p<$ $0.004)$ and IE $(p<0.02)$ than in controls. We found no significant differences in tendon parameters.

Conclusions: The changes in muscle histological properties were those of a chronic rotator cuff tear model reproducible and maintained over time. The effect of denervation in the group with the DT technique pro- 
únicamente en el músculo SE. Siempre y cuando se realice una sección completa de 2 o más tendones del manguito, creando un espacio de al menos $5 \mathrm{~mm}$ entre la huella tendinosa y el extremo de la entesis, asociando la sección del nervio supraescapular, aporta mayores ventajas al modelo animal por la atrofia del músculo SE, mantenida en el tiempo.

Nivel de evidencia: Experimental de tipo I.

Relevancia clínica: Nuestros datos validan el carácter irreversible de la atrofia obtenida consiguiendo el desarrollo de un modelo animal más similar al desgarro crónico humano, especialmente con TD. Ello permitirá realizar futuros estudios mejorando la regeneración de la entesis tras reinserción tendinosa a través del tratamiento coadyuvante con nuevas terapias biológicas.

Palabras clave: Rotura manguito rotador. Modelo experimental. Músculo supraespinoso. Hombro.

\section{Introducción}

La patología de los tendones del denominado manguito rotador del hombro conlleva un gran número de visitas al especialista y elevado coste sanitario anual. A pesar de realizar una técnica exquisita de sutura de desgarros del manguito, según las series existen entre un 20 y un $70 \%$ de fracasos, mayormente debidos a cambios irreversibles tanto en el propio tendón como en el músculo: infiltrado graso, atrofia y baja celularidad, que alteran sus propiedades biomecánicas. Las opciones terapéuticas con las que contamos actualmente para mejorar la calidad de la reparación quirúrgica se hallan aún en fase de desarrollo. Por ello, planteamos como hipótesis de nuestro trabajo mejorar la curación empleando como tratamiento coadyuvante a la cirugía la inoculación directa de células mesenquimales pluripotenciales (MSC), con gran capacidad de diferenciación a diferentes estirpes celulares, tanto en músculo como en entesis (unión tendón-hueso). Para ello, era necesario diseñar en una primera fase un modelo experimental animal que nos permitiera recrear las condiciones patológicas degenerativas del tendón, describiendo la naturaleza de los procesos atróficos y cicatriciales crónicos inducidos mediante tenotomía.

El objetivo del presente trabajo se centra en desarrollar un modelo animal de desgarro cró- duced greater muscle fatty infiltration maintained over time highest ranking in the SE. We found no significant differences in the studied parameters tendon and was indifferent perform a technique or another as long as it was complete section of 2 or more rotator cuff tendons.

Level of evidence: Experimental type I.

Clinical relevance: Our data validate the irreversibility of atrophy obtained achieving the development of a more similar to human chronic tear, especially with DT animal model. This will allow future studies to improve the regeneration of the tendon after reintegration through the adjunctive treatment with new biologic therapies.

Key words: Chronic rotator cuff tears. Chronic rotator cuff tears model. Supraspinatus muscle. Shoulder.

nico del manguito de los rotadores reproducible, lo más similar al encontrado en humanos, que pueda servirnos para investigar, en sucesivas fases, nuevas terapias celulares con expectativas de mejorar el éxito quirúrgico de la reparación.

No existe un modelo ideal único que responda a todas las cuestiones planteadas y se halle exento de limitaciones ${ }^{(1)}$. De entre todas las opciones disponibles para el establecimiento de un modelo animal, seleccionamos el de rata por poseer un arco cerrado, constituido por acromion, coracoides y clavícula, por el cual el tendón del supraespinoso (SE) se desliza a lo largo de todo el movimiento del hombro, similar al humano(2-4).

Este modelo debería también disminuir las limitaciones conocidas de este tipo de modelos experimentales, como por ejemplo la reversibilidad espontánea de los cambios musculares a las 8-12 semanas, la imposibilidad para reproducir el característico infiltrado graso muscular o la aparición de abundante fibrosis en el espacio creado por la tenotomía ${ }^{(3-6)}$. Por ello, deberíamos lograr una mayor degeneración y atrofia tanto tendinosa como muscular que los modelos conocidos. Para evitar adherencias y reversibilidad en el tiempo, asociamos a la tenotomía del SE un desgarro del tendón del músculo infraespinoso (IE) adyacente. Persiguiendo obtener una atrofia muscular e infiltrado graso mantenidos, añadimos también una sección del nervio supraescapular ${ }^{(7)}$. 


\section{Material y métodos}

Para desarrollar un modelo preclínico murino de desgarro crónico del manguito de los rotadores, diseñamos un ensayo aleatorizado con 40 ratas Wistar-Ham hembras, adultas y sanas, de entre 200 y 300 gramos de peso. Se intervinieron ambos hombros de las extremidades anteriores distribuidos en 2 grupos a los que se realizó una técnica simple (TS) de desinserción o una técnica en la que se añadió la denervación (TD) del nervio supraescapular. En el primer grupo de TS se procedió a la desinserción quirúrgica de los tendones de los músculos SE e IE en uno de ambos hombros indistintamente (20 izquierdos, 20 derechos). En el segundo grupo (TD), tras la desinserción de SE e IE, se realizó una denervación añadida del nervio supraescapular (20 y 20). En ambos grupos, se utilizó el lado contralateral como control, realizando solamente incisión cutánea y disección hasta visualizar el manguito, evitando así sesgos y aumentando la potencia estadística con el emparejado.

\section{Criterios de inclusión en el ensayo}

Ratas hembras adultas Wistar-Ham de entre 200 y 300 gramos de peso, con fuerza de agarre de extremidades anteriores normalizada (medida mediante aparato Grip Strength Meter ${ }^{\circledR}$, Bioseb). Los criterios de exclusión fueron la muerte de la rata desde que se produce el desgarro crónico hasta el momento del sacrificio y obtención de las piezas, la desnutrición de la rata con curva negativa de peso en dichas 12 semanas o cualquier complicación ocurrida en la rata durante dicho periodo.

\section{Técnica quirúrgica y anestesia}

El trabajo experimental se desarrolló integramente en las instalaciones del Instituto de Investigación Sanitaria Biodonostia (IISB), que cuenta con todos los permisos e instalaciones para realizar este tipo de proyectos. Los ensayos realizados fueron previamente aprobados por el Comité Ético de Investigación Clínica del Área Sanitaria de Guipúzcoa; acta n.o 11/12 (23/11/2011).

Los animales ingresaron al estabulario una semana antes de la intervención quirúrgica, pe-

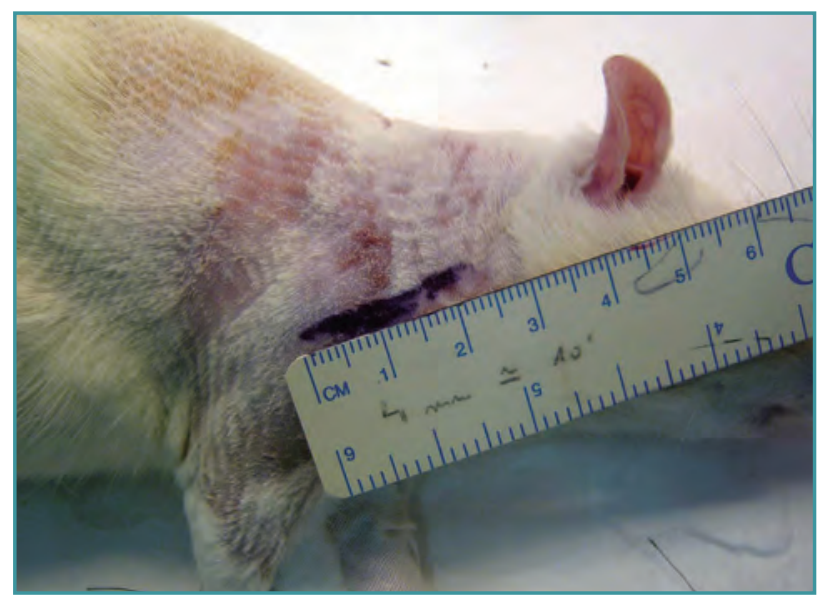

Figura 1. Posición para la intervención en decúbito lateral y ayuda de microscopía óptica; incisión longitudinal de 2 a $3 \mathrm{~cm}$ sobre la articulación escapulohumeral de la extremidad delantera.

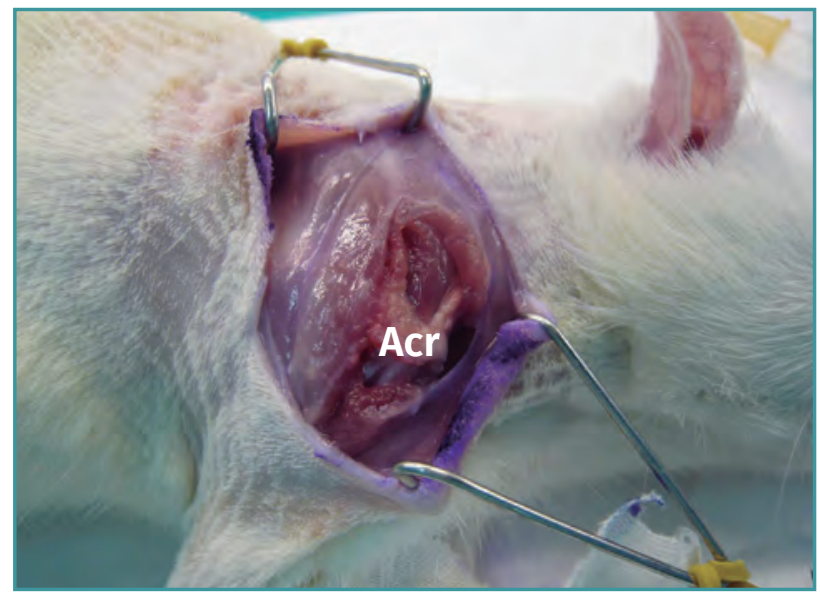

Figura 2. Disección roma del deltoides hasta visualizar el manguito de los rotadores. Acr: acromion.

riodo en que permanecieron en cuarentena, debiendo estar aislados en jaulas de 2 ejemplares cada una según protocolos establecidos de atmósfera estéril. Se realizó la inducción anestésica general mediante una inyección subcutánea de buprenorfina a dosis $0,05 \mathrm{mg} / \mathrm{kg} / 12 \mathrm{~h}$ con dilución 1:5 con suero fisiológico. La anestesia se indujo en cámara de inducción con gas anestésico isoflurano (Forane ${ }^{\circledR}$ ) al $5 \%$ que se mantuvo entre el 2 y el 3\% durante la intervención. Tras el rasurado y la preparación quirúrgica, se inició la intervención en decúbito lateral y con ayuda de microscopía óptica. Se realizó una incisión longitudinal de 2 a $3 \mathrm{~cm}$ sobre la articulación escapu- 


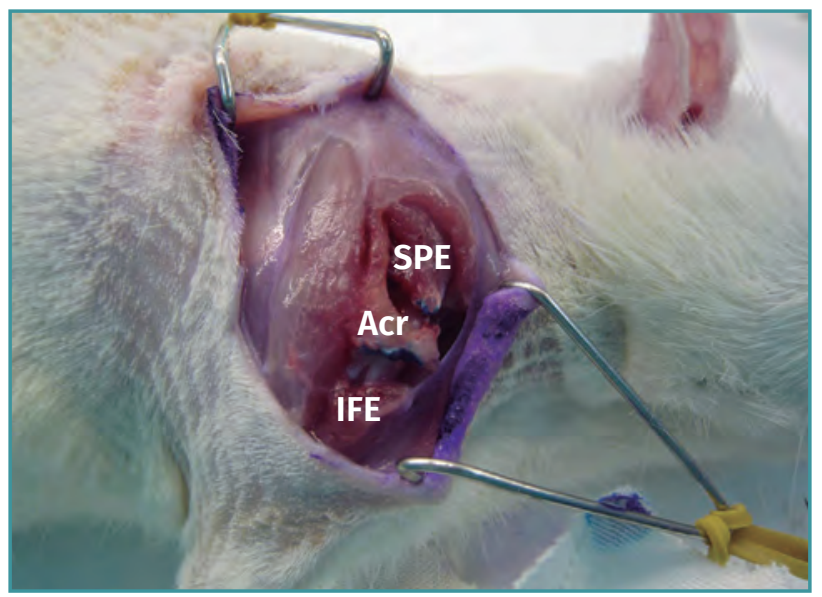

Figura 3. Visión del músculo supraespinoso (SPE) por debajo del arco óseo formado igualmente por acromion, clavícula y coracoides. Adyacente y posterior se encuentra el tendón del músculo infraespinoso (IFE). Acr: acromion.

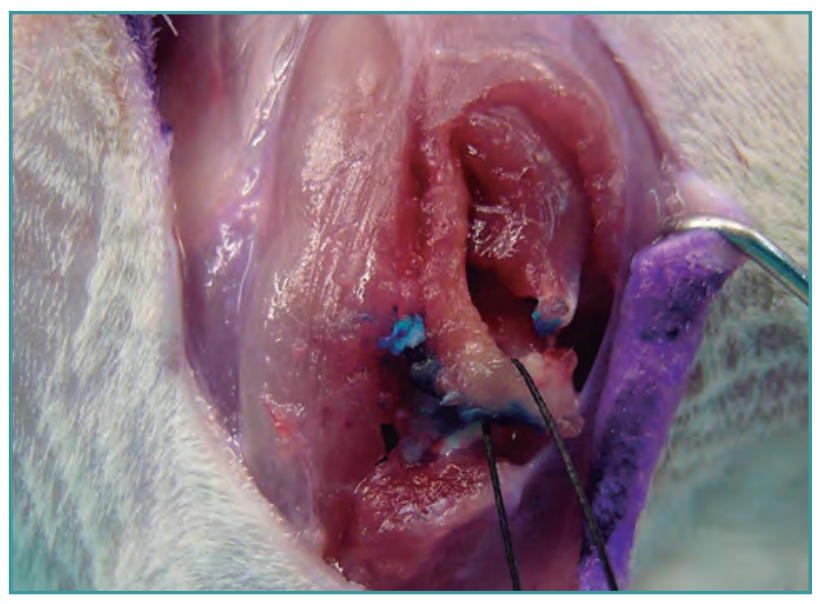

Figura 4. Desinserción completa de ambos tendones creando al menos una retracción tendinosa de $5 \mathrm{~mm}$. SPE: nervio supraescapular.

lohumeral de la extremidad delantera (Figura 1), disección roma de la musculatura deltoidea hasta visualizar el manguito de los rotadores (Figura 2). El tendón SE es rápidamente localizado por debajo del arco óseo similar al humano y formado igualmente por acromion, clavícula y coracoides. Se localizó su inserción en el troquiter humeral. Adyacente y posterior se encuentra el tendón del músculo IE (Figura 3). Se realizó la desinserción completa de ambos tendones comprobando que se creaba al menos una retracción de $5 \mathrm{~mm}$ del muñón tendinoso (Figura 4). Con

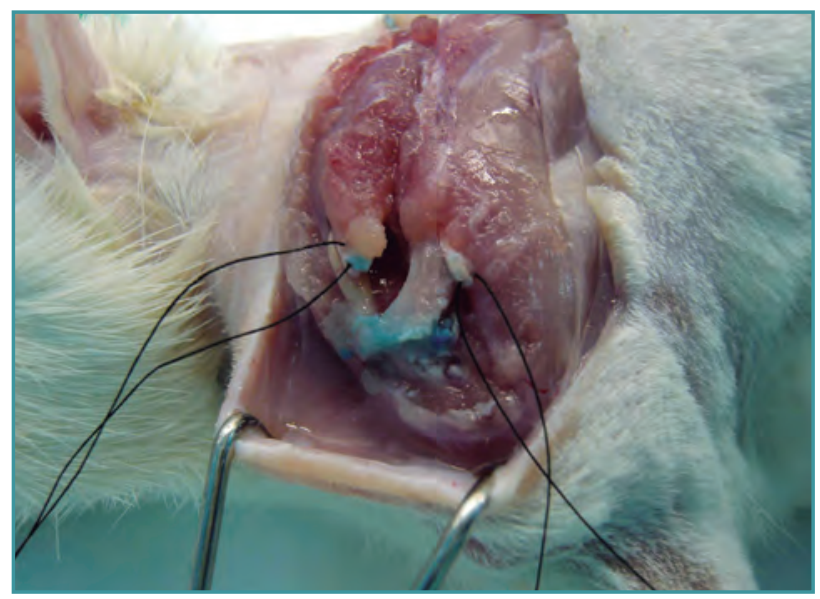

Figura 5. Extremos tendinosos marcados con una sutura monofilamento de 9/0 y denervación del nervio supraescapular.

ello se pretendía evitar la aparición de cicatrizaciones espontáneas. Los extremos tendinosos se dejaban marcados con una sutura de monofilamento 9/0. Se asoció la denervación del nervio supraescapular, localizándolo por debajo de la porción central muscular SE (Figura 5). Por último, se practicó reconstrucción del deltoides y cierre de la piel con suturas reabsorbibles de $5 / 0$ y $3 / 0$, respectivamente.

\section{Curso postoperatorio}

El animal se instaló en su correspondiente jaula aislada y libre de patógenos, permitiéndole libre movilidad en la misma. Diariamente, se evaluó el consumo de agua y alimentos, el estado de la herida y la movilidad espontánea. La analgesia postoperatoria se aplicó con buprenorfina (Buprex ${ }^{\circledR}$ ) a demanda. Los ejemplares fueron sacrificados con administración de $\mathrm{CO}_{2}$ durante 10 minutos, según las tandas designadas a las 4, 8 y 12 semanas posteriores a la intervención para realizar la extracción y el estudio de sus respectivos juegos miotendinosos.

\section{Análisis histológico macro- y microscópico}

Las piezas musculares completas, extraídas en los diferentes puntos en el tiempo descritos, 
fueron pesadas y medidas volumétricamente e inmediatamente incluidas en formol. Para la evaluación histológica, las piezas se sometieron a un proceso de deshidratación con diferentes alcoholes e inclusión en parafina a $60^{\circ} \mathrm{C}$ para realizar posteriores cortes longitudinales y transversales de 5 a 8 micras de grosor y permitir la aplicación de las tinciones adecuadas. Se realizó estudio y lectura de las muestras con ayuda de microscopía óptica y software Fiji-Imagej) ${ }^{\circledR}$ (análisis estereológico). Se revisaron entre 3 y 5 muestras de cada pieza.
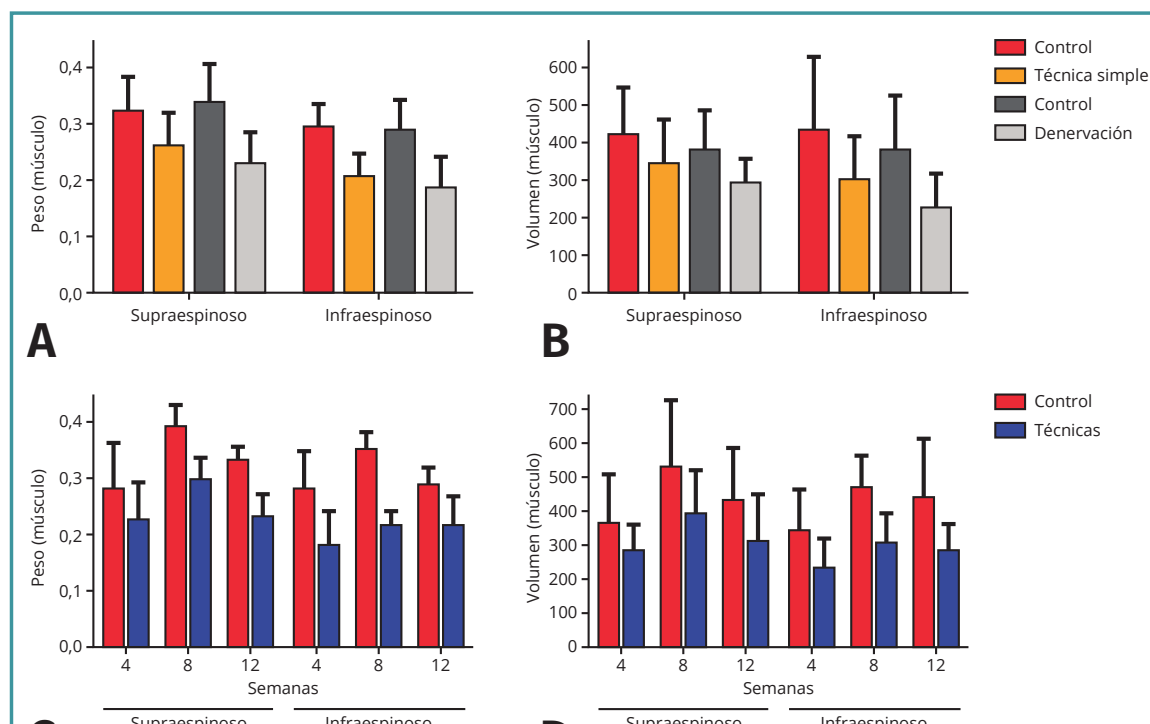

C

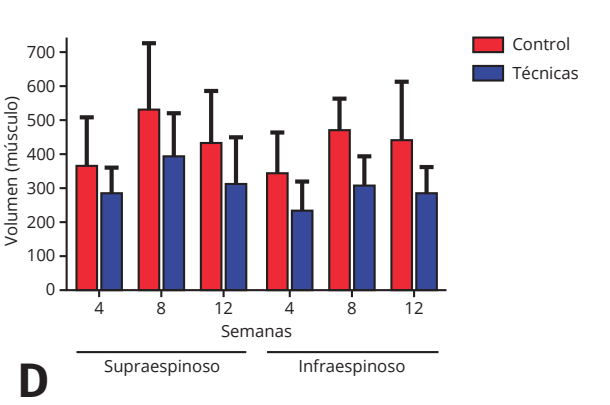

Figura 6. Resultados del examen macroscópico del músculo. Muestra los resultados de disminución de peso (A) y de volumen (B) comparando la técnica simple con su control y con la técnica de denervación y su control. Ese efecto de disminución se mantiene en el tiempo tanto en relación con el tiempo (C) como con el volumen (D).

\section{Análisis muscular y tendinoso}

Con hematoxilina-eosina (HE) se analizó la celularidad, la tipificación y la medición de la organización del colágeno. Para observar y cuantificar la grasa intramuscular se aplicó tricrómico de Masson (TM). Las áreas colectivas tisulares obtenidas fueron evaluadas según un método estereológico cuantitativo con ayuda de software Fiji-Imagej ${ }^{\circledR}$ y escáner.

En cuanto a la evaluación de la entesis, previo procesado y decalcificado rápido de 36 horas, se analizó en un primer momento su estructura y celularidad con tinciones de HE y TM para valorar la formación de nueva estructura fibrocartilaginosa.

\section{Análisis estadístico}

Se utilizaron métodos estadísticos no paramétricos para análisis de datos, comparando los datos diferentes a la normalidad. Se utilizó el test de Kruskal-Wallis para comparar los grupos de distribución. Todos los datos se expresaron como media \pm desviación estándar. Un valor de $p<0,05$ indicaba una diferencia estadísticamen- te significativa. Para el análisis se utilizó el software GraphPad Prism ${ }^{\circledR}$ (versión 5).

\section{Resultados}

\section{Evaluación macroscópica}

Observamos que la realización de la intervención quirúrgica en ambas extremidades superiores no comprometía la vitalidad de los animales. Aunque inicialmente las ratas intervenidas presentaban una tendencia a perder peso (aproximadamente un $4 \%$ de su peso inicial), a las 2 semanas postintervención ya recuperaban los valores de su peso preoperatorio e incluso seguían ganado peso. Ciframos esta ganancia en una media del $17 \%$ sobre el peso inicial.

Al comparar, el peso y el volumen de los músculos SE e IE del hombro intervenido respecto al hombro control para cada técnica, TS y TD, observamos que, independientemente del músculo y la técnica utilizada, el hombro intervenido presentaba una disminución significativa $(p<0,01)$ de ambos parámetros; incluso, llegan- 
Primeras fases del desarrollo de un modelo experimental [...]

Tabla 1. Resultados microscópicos

\begin{tabular}{|c|c|c|c|c|}
\hline & $\begin{array}{l}\text { Grupo intervención } \\
\text { media (DE) }\end{array}$ & Grupo control media (DE) & $\begin{array}{l}\text { Promedio diferencia ajustado por } \\
\text { peso al sacrificio (IC 95\%) }\end{array}$ & $\mathbf{p}$ \\
\hline \multicolumn{5}{|c|}{ Hombro intervenido derecho } \\
\hline \multicolumn{5}{|c|}{ Supraespinoso derecho intervenido } \\
\hline Peso (gramos) & $0,24(0,06)$ & $0,29(0,03)$ & $-0,10(-0,16 ;-0,04)$ & 0,002 \\
\hline Volumen $\left(\mathrm{mm}^{3}\right)$ & $339,60(132,29)$ & $446,20(109,62)$ & $-191,01(-354,78 ;-27,23)$ & 0,02 \\
\hline \multicolumn{5}{|c|}{ Infraespinoso derecho intervenido } \\
\hline Peso (gramos) & $0,20(0,06)$ & $0,25(0,04)$ & $-0,07(-0,12 ;-0,01)$ & 0,02 \\
\hline Volumen $\left(\mathrm{mm}^{3}\right)$ & $287,87(77,62)$ & $379,35(136,62)$ & $-141,28(-265,76 ;-16,80)$ & 0,03 \\
\hline \multicolumn{5}{|c|}{ Hombro intervenido izquierdo } \\
\hline \multicolumn{5}{|c|}{ Supraespinoso izquierdo intervenido } \\
\hline Peso (gramos) & $0,25(0,06)$ & $0,28(0,04)$ & $-0,06(-0,13 ; 0,01)$ & 0,08 \\
\hline Volumen $\left(\mathrm{mm}^{3}\right)$ & $333,53(112,48)$ & $464(92,43)$ & $-178,22(-319,55 ;-36,88)$ & 0,02 \\
\hline \multicolumn{5}{|c|}{ Infraespinoso izquierdo intervenido } \\
\hline Peso (gramos) & $0,19(0,05)$ & $0,29(0,06)$ & $-0,14(-0,21 ;-0,07)$ & 0,001 \\
\hline Volumen $\left(\mathrm{mm}^{3}\right)$ & $255,91(86,73)$ & $372,75(57,43)$ & $-121,29(-231,60 ;-10,98)$ & 0,03 \\
\hline
\end{tabular}

do a ser dicha diferencia más significativa con la TD. Al realizar el estudio de ambos parámetros en distintos momentos del seguimiento $(4,8 \mathrm{y}$ 12 semanas poscirugía), el patrón observado fue muy similar. Es decir, en los hombros intervenidos, tanto con la TS como con la TD, el peso y el volumen de los músculos SE e IE son inferiores que en los hombros control, efecto que se mantiene en el tiempo. En el músculo SE sí observamos diferencias estadísticamente significativas $(p<0,03)$ al comparar ambas técnicas quirúrgicas durante el periodo de 8 y 12 semanas (Figura 6 y Tablas 1 y 2 ).

\section{Análisis microscópico del músculo}

En relación con el análisis de infiltrado graso intramuscular respecto a sus controles con ambas técnicas, hallamos que los hombros intervenidos presentaban un aumento constante y progresivo del porcentaje de grasa muscular. Este porcentaje llegaba a ser significativo $(p<0,01)$ cuando el músculo SE es intervenido mediante la TD (Figura 7A). A su vez, al analizar el porcentaje de grasa en distintos tiempos en ambos músculos, observamos que a las 12 semanas postintervención mediante la TD el porcentaje de infiltrado es significativamente mayor en los músculos SE $(p<0,004)$ e IE $(p<0,02)$ que en sus controles (Figura 7B). Además, mediante la tinción de HE determinamos que en el músculo SE la cantidad de grasa intramuscular aumenta cuanto mayor es el tiempo transcurrido desde la cirugía hasta el sacrificio y la extracción de la pieza.

En referencia al porcentaje de fibrosis muscular respecto a la superficie muscular total, observamos que en función de la técnica utilizada el porcentaje de fibrosis variaba independientemente del tiempo transcurrido. Las diferencias más significativas las detectamos en el músculo IE, concretamente a las 12 semanas poscirugía con la TD (Figura 8 A y B). No obstante, en el porcentaje de fibrosis del músculo SE no encontramos ningún cambio. 
Tabla 2. Resultados microscópicos

\begin{tabular}{|c|c|c|c|c|}
\hline & $\begin{array}{l}\text { Técnica simple } \\
\text { media (DE) }\end{array}$ & $\begin{array}{c}\text { Técnica con denervación } \\
\text { media (DE) }\end{array}$ & $\begin{array}{l}\text { Promedio diferencia ajustado por } \\
\text { peso al sacrificio (IC 95\%) }\end{array}$ & $\mathbf{p}$ \\
\hline \multicolumn{5}{|c|}{ Total } \\
\hline \multicolumn{5}{|l|}{ Supraespinoso } \\
\hline Peso (gramos) & $-0,06(0,07)$ & $-0,11(0,08)$ & $-0,04(-0,10 ;-0,01)$ & 0,1 \\
\hline Volumen $\left(\mathrm{mm}^{3}\right)$ & $-76,72(153,14)$ & $-136,10(152,53)$ & $-59,11(-173,22 ; 55)$ & 0,3 \\
\hline \multicolumn{5}{|l|}{ Infraespinoso } \\
\hline Peso (gramos) & $-0,09(0,06)$ & $-0,10(0,07)$ & $-0,02(-0,06 ; 0,03)$ & 0,46 \\
\hline Volumen $\left(\mathrm{mm}^{3}\right)$ & $-88,13(121,81)$ & $-154,00(91,63)$ & $-65,44(-145,12 ; 14,24)$ & 0,1 \\
\hline \multicolumn{5}{|c|}{4 semanas } \\
\hline \multicolumn{5}{|l|}{ Supraespinoso } \\
\hline Peso (gramos) & $-0,06(0,12)$ & $-0,04(0,12)$ & $0,02(-0,23 ; 0,26)$ & 0,87 \\
\hline Volumen $\left(\mathrm{mm}^{3}\right)$ & $-137,5(135,82)$ & $-24,38(116,31)$ & $112,63(-136,08 ; 361,33)$ & 0,3 \\
\hline \multicolumn{5}{|l|}{ Infraespinoso } \\
\hline Peso (gramos) & $-0,13(0,03)$ & $-0,07(0,11)$ & $0,05(-0,11 ; 0,22)$ & 0,43 \\
\hline Volumen $\left(\mathrm{mm}^{3}\right)$ & $-108,25(79,07)$ & $-102,25(91,12)$ & $5,15(-150,92 ; 161,22)$ & 0,94 \\
\hline \multicolumn{5}{|c|}{8 semanas } \\
\hline \multicolumn{5}{|l|}{ Supraespinoso } \\
\hline Peso (gramos) & $-0,06(0,03)$ & $-0,12(0,05)$ & $-0,06(-0,13 ; 0,01)$ & 0,07 \\
\hline Volumen $\left(\mathbf{m m}^{3}\right)$ & $-51,80(203,68)$ & $-213,30(103,28)$ & $-156,10(-484,73 ; 172,53)$ & 0,3 \\
\hline \multicolumn{5}{|l|}{ Infraespinoso } \\
\hline Peso (gramos) & $-0,10(0,03)$ & $-0,14(0,05)$ & $-0,04(-0,10 ; 0,02)$ & 0,17 \\
\hline Volumen $\left(\mathrm{mm}^{3}\right)$ & $-102,4(126,77)$ & $-146,25(95,18)$ & $-110,58(-291,97 ; 70,81)$ & 0,19 \\
\hline \multicolumn{5}{|c|}{12 semanas } \\
\hline \multicolumn{5}{|l|}{ Supraespinoso } \\
\hline Peso (gramos) & $-0,06(0,05)$ & $-0,14(0,05)$ & $-0,08(-0,15 ;-0,01)$ & 0,03 \\
\hline Volumen $\left(\mathrm{mm}^{3}\right)$ & $-59,79(126,77)$ & $-146,25(88,86)$ & $-81,10(-237,97 ; 75,77)$ & 0,28 \\
\hline \multicolumn{5}{|l|}{ Infraespinoso } \\
\hline Peso (gramos) & $-0,05(0,06)$ & $-0,09(0,03)$ & $-0,05(-0,11 ; 0,02)$ & 0,14 \\
\hline Volumen $\left(\mathrm{mm}^{3}\right)$ & $-63,43(149,21)$ & $-137,58(71,29)$ & $-52,80(-207,41 ; 101,81)$ & 0,46 \\
\hline
\end{tabular}

En cuanto al diámetro de las fibras, no observamos diferencias significativas independientemente del músculo y de la técnica utilizada (Figu- ra 9). El número de fibras musculares por campo tras la intervención tampoco demuestra diferencias significativas. 


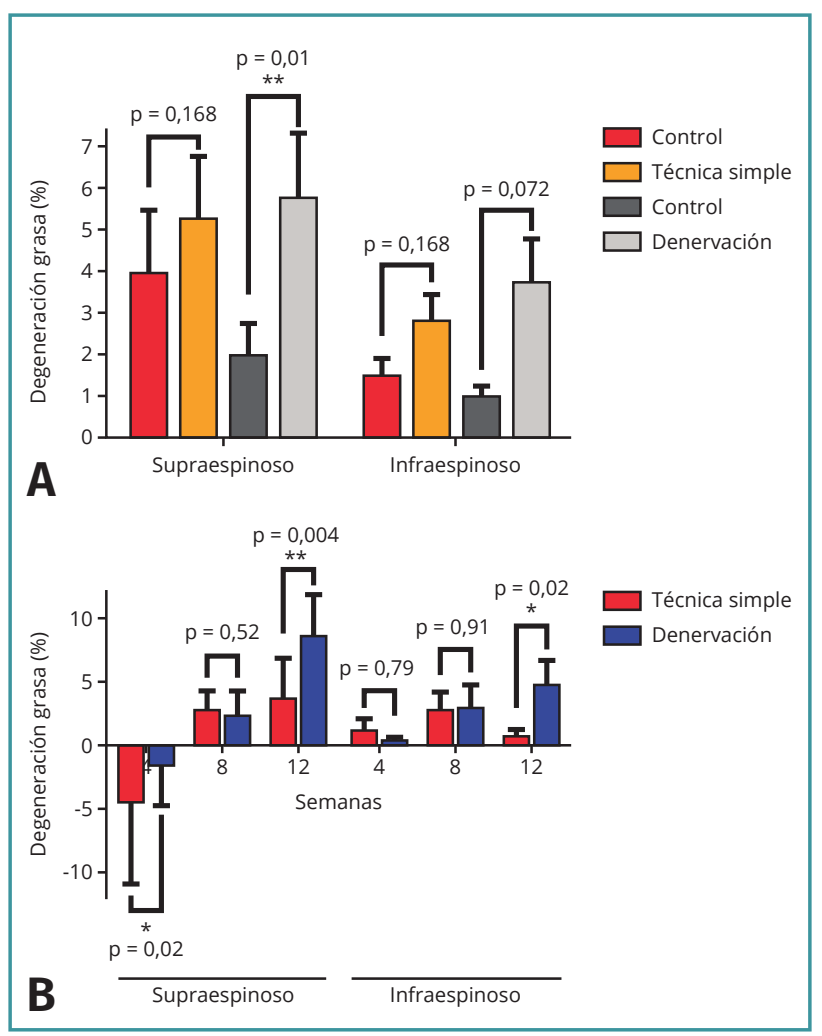

Figura 7. Resultados del análisis de infiltrado graso intramuscular. Según estos resultados, ambas técnicas producen mayor infiltrado graso que los controles y la técnica con denervación produce la diferencia más significativa (A). La mayor diferencia entre técnicas se produce a las 12 semanas (B).

\section{Evaluación microscópica de los tendones}

En general no encontramos diferencias significativas en los parámetros tendinosos estudiados. Es decir, no hay diferencias entre la realización de una u otra técnica.

Pudimos apreciar que en la porción tendinosa de la pieza intervenida el porcentaje de tejido fibroso es significativamente mayor que su correspondiente control, independientemente de la técnica utilizada (Figura 10 A y B).

El ángulo de la alineación de las fibras representa la pérdida de orientación de las mismas. En cuanto a dicho parámetro, los tendones intervenidos presentan una mayor desorientación que sus respectivos controles independientemente de la técnica utilizada, pero sin llegar a la significación estadística en ningún caso (Figura 11).

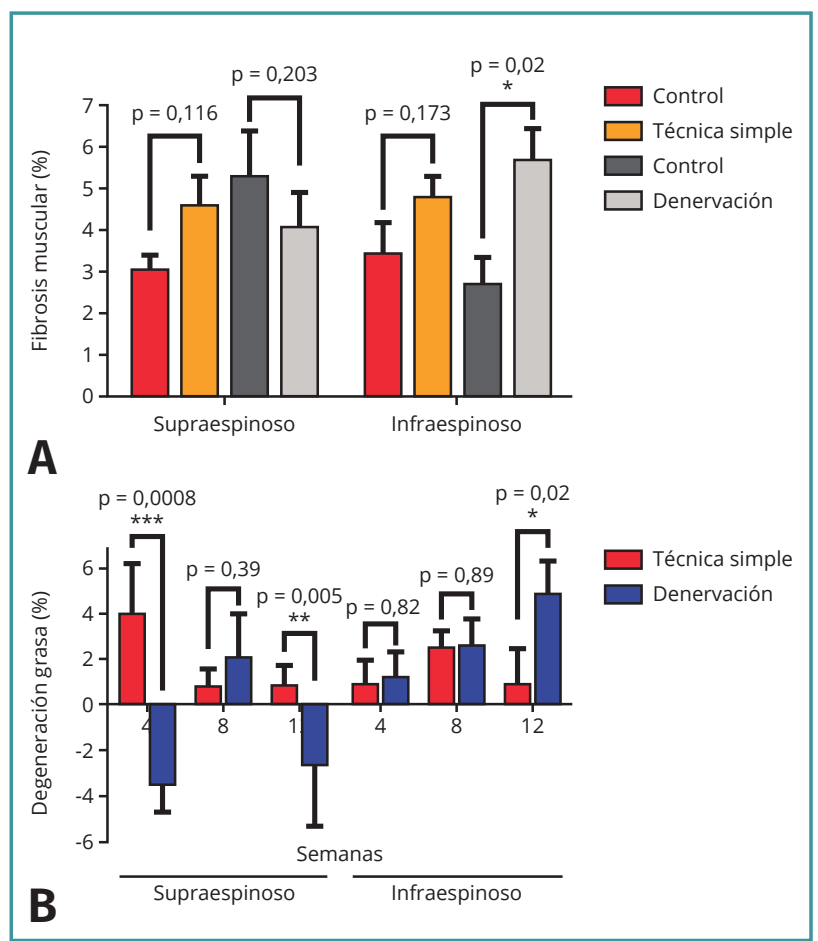

Figura 8. Resultados del porcentaje de fibrosis muscular respecto a la superficie muscular total. La fibrosis muscular aumenta en el músculo infraespinoso pero no en el supraespinoso (A). La significación estadística solo aparece con la técnica de denervación en el músculo infraespinoso a partir de las 12 semanas (A-B).

\section{Discusión}

En la práctica clínica diaria encontramos una alta tasa de recidivas posquirúrgicas de lesiones crónicas y masivas del manguito rotador de hombro, persistiendo dolor e incapacidad debido a su escasa posibilidad de cicatrización a pesar de una correcta técnica quirúrgica. El propósito de este estudio era perfeccionar los modelos animales de desgarro del manguito rotador en animal pequeño existentes, insistiendo en el carácter de cronicidad y ruptura masiva. Con nuestros resultados hemos logrado acercarnos de la manera más reproducible posible a los cambios patológicos crónicos observados en humanos (sobre todo al grado de infiltrado graso y de atrofia muscular característicos de las etapas avanzadas)(2,3). Ello nos permitirá en un futuro próximo ensayar nuevas terapias biológicas coadyuvantes a la cirugía 


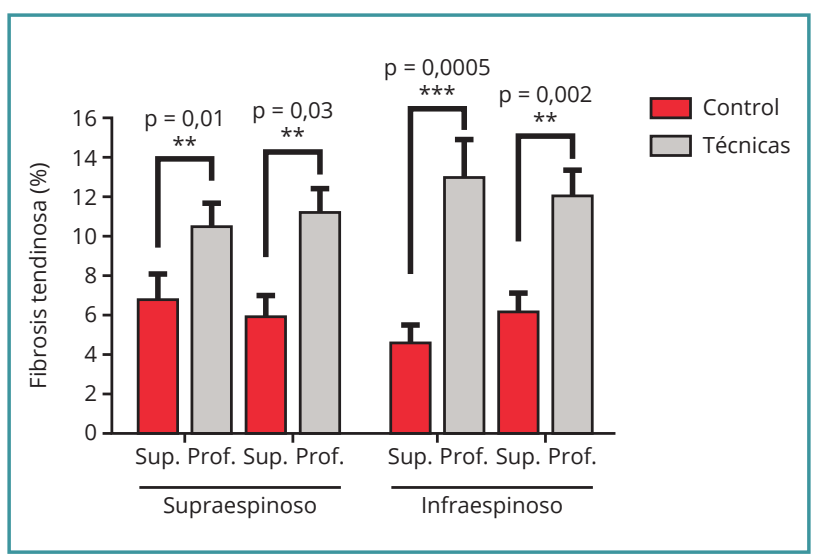

Figura 9. Resultados de la evaluación microscópica de los tendones. El porcentaje de fibrosis en el tendón aumenta significativamente tras la técnica, observando la mayor significación a las 8 semanas y siendo mayor en el músculo infraespinoso que en el supraespinoso.

para mejorar significativamente los resultados actuales.

En este trabajo, seleccionamos, como propuso Soslowsky et al.(4), un modelo en rata por su gran similitud anatómica ósea al humano, a pesar de sus diferentes funcionalidades de las extremidades anteriores. Este modelo es, sin embargo, mucho más parecido al humano que el de conejos, corderos, ovejas o perros. Siguiendo técnicas descritas ${ }^{(5,6)}$, realizamos desinserciones agudas abiertas de los tendones del manguito rotador para luego, a continuación, cerrar y analizar sus características a lo largo del tiempo hasta encontrar el momento en que aparecen signos significativos de cronicidad sin detectar reversibilidad de los datos encontrados. El pico máximo de atrofia muscular secundario al desgarro agudo se estableció en torno a las 4 semanas, por la alta tasa de cicatrización y la tendencia a la formación de adherencias a las 8 y 12 semanas, respectivamente, como en otros trabajos simi- lares $^{(5,6)}$. Por este motivo, se decidió realizar un seguimiento suficiente hasta la semana 12 para poder observar y cuantificar los aspectos de cronicidad y reversibilidad a más largo plazo.

Sobre los modelos conocidos, hemos realizado varias modificaciones significativas para asegurar la reproducibilidad y cronicidad de las lesiones e intentar controlar las limitaciones descritas en anteriores estudios ${ }^{(5-8)}$. Así, en vez de una desinserción única del tendón SE, empleamos el desgarro combinado de los tendones de los músculos SE e IE. Con ello, la lesión se asemejaba más a las lesiones complejas humanas. Se pretendía crear además un defecto crítico con retracción suficiente de al menos $5 \mathrm{~mm}$ y así poder evitar la formación de adherencias y reducir el índice de cicatrizaciones espontáneas ${ }^{(5,8)}$. También hemos añadido al modelo una sección del nervio supraescapular. Con ello pretendíamos aumentar y prolongar el grado de atrofia muscular y de infiltrado graso, características habituales de las roturas crónicas en humanos. También, como aportación novedosa, estudiamos los datos emparejados realizando un control negativo (sham surgery) en el lado contralateral llegando al manguito rotador a través del deltoides. Con ello, a la vez de aumentar la potencia estadística del ensayo, pudimos comprobar la viabilidad y las

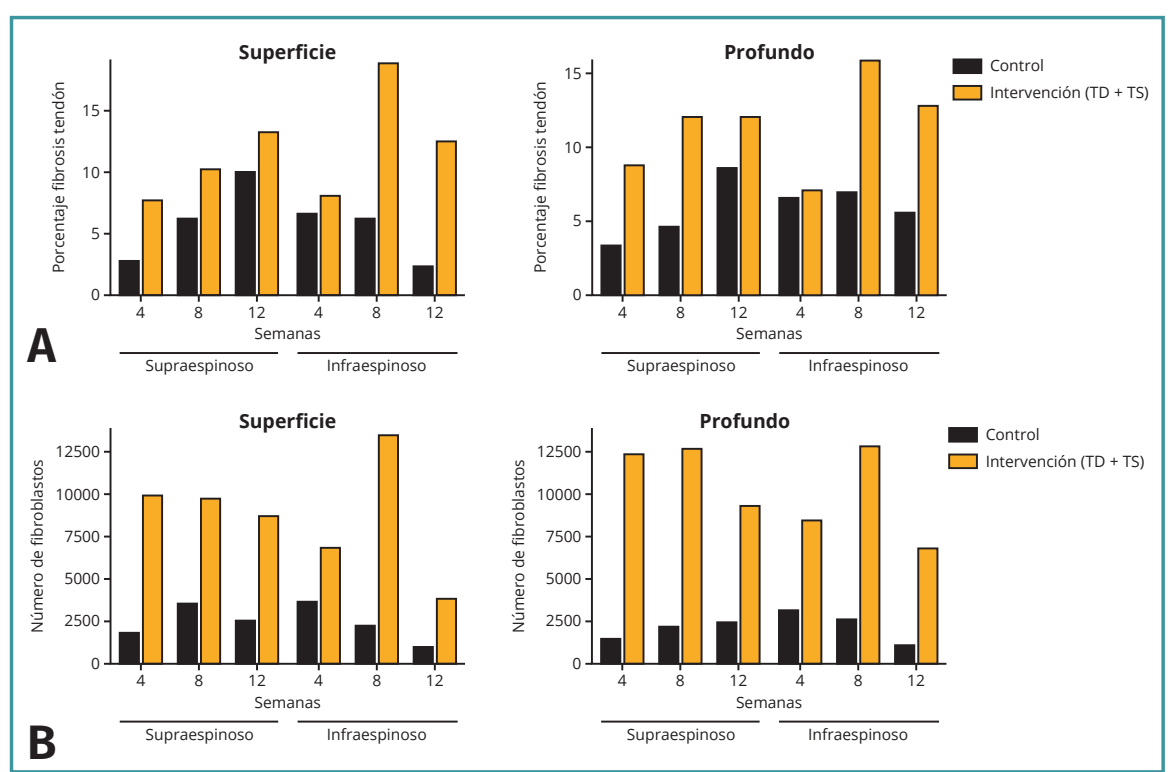

Figura 10. Resultados de la evaluación microscópica de los tendones. A: en la porción tendinosa de la pieza intervenida (TD y TS) el porcentaje de tejido fibroso es significativamente mayor que su correspondiente control en los diferentes tiempos; B: el número de fibroblastos es mayor en tendones intervenidos (intervención) frente a controles en todos los puntos de control a lo largo del tiempo. 


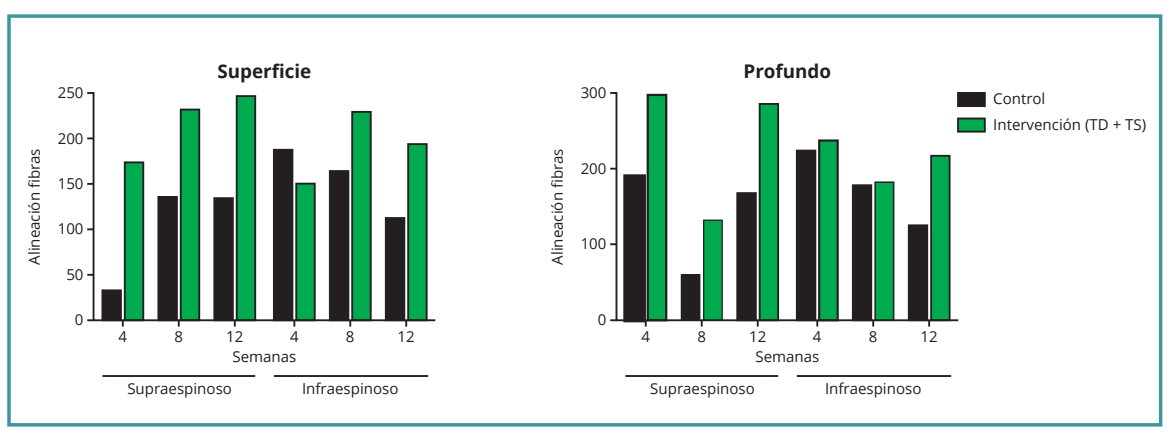

Figura 11. Resultados de la evaluación microscópica de los tendones. El ángulo de alineación de las fibras representa la pérdida de orientación de las mismas. En cuanto a dicho parámetro, los tendones intervenidos presentan una mayor desorientación que sus respectivos controles.

implicaciones de la cirugía simultánea en ambas extremidades anteriores, entre ellas, la existencia de hipertrofia contralateral por sobreuso. Y, por último, realizamos un análisis de las propiedades histológicas del tendón, entendiéndolo como una unidad junto con su músculo correspondiente.

Encontramos datos en el examen histológico que soportan el hecho de haber conseguido obtener el carácter crónico pretendido de las lesiones provocadas. Así, analizando los resultados macroscópicos del estudio podemos corroborar que la disminución de parámetros como peso y volúmenes son mantenidos y progresivos en el tiempo hasta las 12 semanas, tanto realizando la técnica TS como la TD en ambos músculos. Estos datos indican que la atrofia muscular por desuso se perpetúa crónicamente. En los casos control no se produce una apreciable hipertrofia contralateral que haga pensar en un sesgo de estos resultados. Además, la diferencia de peso es mayor a las 8 semanas tras el desgarro en ambos músculos, equivalente en las 2 técnicas, TS y TD. Estas diferencias se reducen solo mínimamente en el grupo de 12 semanas (Figura 6 y Tablas 1 y 2).

La presencia de infiltrado graso muscular inestable e irregular es uno de los mayores problemas referidos por diversos autores en los diferentes modelos animales descritos ${ }^{(3-8)}$. Esta es una diferencia importante del patrón de lesión en los humanos, en los que se produce un infiltrado graso mantenido en los desgarros crónicos irreversibles. Analizando nuestros resultados, el infiltrado graso hallado es mayor y constante en ambos músculos, SE e IE, en los casos de las series de desgarro (TS y TD) frente a las series de control. La diferencia en los músculos SE sometidos a técnica TD es muy significativa cuanto mayor es el tiempo de seguimiento (12 semanas). Esta es, por tanto, una diferencia importante en relación con otros modelos anteriores que no mantienen o no logran provocar atrofia grasa en ninguna de las piezas musculares estudiadas, incluso en todo el tiempo de seguimiento(5,8). Nuestros datos sobre infiltrado graso muscular validan el carácter irreversible de la atrofia obtenida, añadiendo valor al modelo animal desarrollado.

En cambio, los datos encontrados en cuanto al porcentaje de fibrosis muscular provocado no son tan consistentes. Si bien en el músculo IE dicho porcentaje de fibrosis muscular aumenta tras la sección, incluso más con la técnica DT, no hemos podido valorar estos datos en el músculo SE. Quizás ello pueda ser debido a que el tamaño de la muestra sea pequeño para demostrar los verdaderos efectos de fibrosis en el SE (Figura 8).

En nuestra hipótesis postulábamos que la atrofia muscular conllevaría un descenso del diámetro de la fibra en los músculos seccionados atróficos, además de un descenso de las fibras musculares. Pero los resultados nos han demostrado que no hay diferencias en el diámetro de la fibra ni en el número de ellas, independientemente de la técnica realizada. Otros autores invalidan la medida del diámetro de las fibras, así como de la densidad de los núcleos existentes en ellas como medida de cuantificación de la atrofia muscular, llegando a la conclusión de que no es un método adecuado(9). Ello sería debido a que la disminución del área producida por la sección (al estar sometida a menor tensión) puede hacer que el diámetro de las fibras aumente en músculos atróficos, con lo cual se invalidaría la medición.

En el presente modelo se produce, de forma no deseada (apenas existente en humanos), una proliferación de tejido cicatricial entre el extremo del tendón seccionado y las estructuras adyacentes, más en el caso del tendón SE. Esta proliferación cicatricial hallada es similar a la encontrada en los otros modelos descritos ${ }^{(5-9)}$. Pero en todos 
los casos de nuestro modelo el tejido cicatricial hallado no llegó a alcanzar el punto de inserción original de los tendones y no constituyó, por ello, un impedimento para que se desarrollase el proceso de carácter crónico descrito.

\section{Limitaciones del estudio}

Es cierto que el animal seleccionado para realizar el presente trabajo no ofrece la misma cinemática articular ni habilidades con sus extremidades anteriores similares a las de los humanos ${ }^{(5,9)}$, pero sí lo hace de manera más acorde que otros modelos animales descritos ${ }^{(4)}$. Quizás, otra limitación la constituye el hecho de dividir todo el tamaño de la muestra utilizada en cada etapa del análisis (4, 8 y 12 semanas) pudiendo limitar el adquirir una adecuada potencia estadística final. Por ello, para disminuir la posible variabilidad, hemos utilizado muestras emparejadas por cada grupo con su control en el mismo individuo. Partimos de un daño agudo realizado mediante una sección quirúrgica, no recreando de forma ortodoxa las condiciones habituales de rotura masiva del manguito rotador. Además, reducido por la sección de ambos tendones, SE e IE, podemos observar cicatrizaciones espontáneas que sesgarían nuestros resultados, como en otros modelos descritos ${ }^{(5,6,9)}$. Finalmente, únicamente obtuvimos datos tras el sacrificio del animal. En futuros estudios se podrían analizar, también in vivo, las diferencias en habilidad o fuerza del modelo de desgarro crónico del manguito. A pesar de las limitaciones descritas, con este estudio se demuestra la validez del modelo desarrollado de desgarro crónico masivo del manguito rotador en animal pequeño. Así, se podrá aplicar a posteriores ensayos preclínicos de desarrollo de nuevas terapias biológicas ${ }^{(10-13)}$. Sería de gran utilidad completar este trabajo mediante estudios que aporten datos sobre la fuerza y la habilidad de extremidades anteriores tras sección tendinosa, complementando y permitiendo corroborar la validez del modelo murino descrito.

\section{Conclusiones}

Los cambios provocados en las propiedades histológicas musculares a lo largo del presente trabajo son los propios de un modelo crónico de desgarro del manguito rotador. Representan así un modelo fiable, reproducible y mantenido que permite el estudio de diferentes terapias biológicas coadyuvantes en animal pequeño. El efecto de la denervación añade un mayor infiltrado graso muscular, aunque únicamente en el SE. Se demuestra que, siempre y cuando se realice una sección completa de 2 o más tendones del manguito creando un espacio de al menos $5 \mathrm{~mm}$ entre la huella tendinosa y el extremo de la entesis, realizar una denervación asociada por sección del nervio supraescapular aporta mayores ventajas a dicho modelo animal por la atrofia del SE mayormente mantenida en el tiempo.

\section{Agradecimientos}

Agradecemos toda la ayuda prestada por el personal administrativo y de investigación del Instituto de Investigación Sanitaria Biodonostia y especialmente de los doctores Ander Izeta Permisan (Director de la Unidad de Ingeniería Tisular) y Carlos San José Marqués (Director del animalario). Su ayuda ha sido imprescindible para poder desarrollar el presente trabajo.

\section{Financiación}

Este trabajo ha sido financiado con las becas otorgadas por: 1) el Departamento de Innovación y Sociedad del Conocimiento de la Diputación Foral de Guipuzkoa (BOG no 246 de 27/12/2010), Orden Foral $53 / 2011$ y 2) por la Fundación Española de Artroscopia en su convocatoria de 26 abril de 2013.

\section{Conflicto de intereses}

Los autores declaran no tener ningún conflicto de intereses en la realización y preparación del presente trabajo.

\section{Responsabilidades éticas}

Los autores declaran que los procedimientos seguidos se conformaron a las normas éticas del comité de experimentación humana responsable y de acuerdo con la Asociación Médica Mundial y la Declaración de Helsinki. 


\section{Bibliografía}

1. Muschler GF, Vivek P, Patterson TH, Wenke JC, Hollinger J. The design and use of animal models for translational research in bone tissue engineering and regenerative medicine. Tissue Eng. 2010;16:125-43.

2. Maffulli N, Longo UJ, Franceschi F, Rabitti C, Denaro V. Movin and Bonar scores assess the same characteristics of tendon histology. Clin Orthop Related Res. 2008;466:1605-11.

3. Longo UG, Franceschi F, Ruzzini L, Rabitti C, Morini S, Maffulli N, Denaro V. Histopathology of the supraespinatus tendon in rotator cuff tears. Am J Sports Med. 2008;36:533-8.

4. Soslowsky LJ, Carpenter JE, DeBano CM, Banerji I, Moalli MR. Development and use of an animal model for investigations on rotator cuff disease. J Shoulder Elbow Surg. 1996;5:383-92.

5. Barton ER, Gimbel JA, Williams GR, Soslowsky LJ. Rat supraespinatus muscle atrophy after tendon detachment. J Orthop Res. 2005;23:259-65.

6. Gimbel JA, Van Kleunen JP, Mehta S, Perry SM, Williams GR, Soslowsky LJ. Supraespinatus tendon organizational and mechanical properties in a chronic rotator cuff tear animal model. J Biomechanics. 2004;37:739-49.
7. Cho E, Zhang Y, Pruznak A, Kim HM. Effect of tamoxifen on fatty degeneration and atrophy of rotator cuff muscles in chronic rotator cuff tear: an animal model study. J Orthop Res. 2015;33:1846-53.

8. Dourte LM, Perry SM, Getz CL, Soslowsky LJ. Tendon properties remain altered in a chronic rat rotator cuff model. Clin Orthop Relat Res. 2010;468:1485-92.

9. Liu X, Manzano G, Kim HT, Feeley BT. A rat model of massive rotator cuff tears. J Orthop Res. 2011;29:588-95.

10. Wang QW, Chen ZL. Mesenchymal stem cells differentiate into tenocytes by bone morphogenetic protein (BMP) 12 gene transfer. J Biosci Bioeng. 2005;100: 418-22.

11. Rodeo SA. Biologic augmentation of rotator cuff tendon repair. J Shoulder Elbow Surg. 2007;16(5 Sup$\mathrm{pl)}:$ S191-7.

12. Beason DP, Connizzo BK, Dourte LM, Mauck RL, Soslowsky LJ, Steinberg DR, Bernstein J. Fiber aligned polymer scaffolds for rotator cuff repair in a rat model. J Shoulder Ebow Surg. 2012;21:245-50.

13. Davis ME, Korn MA, Gumucio JP, Harning JA, Saripalli AL, Bedi A, Medias CL. Simvastatin reduces fibrosis and protects against muscle weakness after massive rotator cuff tear. J Shoulder Elbow Surg. 2015;24:280-7. 